\title{
Genesis of a pulsar's planets
}

SiR - Wolszczan and Frail ${ }^{1}$ have discovered at least two planets in nearly circular orbits with periods of 66.6 and 98.2 days around PSR1257+12. We propose that these planets formed before the pulsar, originally orbiting a close binary star at a distance of a few $\mathrm{AU}$. The primary star of the binary formed a neutron star. The secondary first acted as an extra mass, to ensure that less than half the system mass was lost in the supernova explosion and the binary and the planets remained bound. The secondary was destroyed in a subsequent phase of mass transfer. The mass flow concomitant with this destruction engulfed the planets, causing their orbits to shrink and circularize. This scheme has the advantage over existing ones that (1) its progenitors are probably quite abundant; (2) it can explain planets around old, recycled pulsars; and (3) the number of planets is not limited to one per pulsar.

Specifically, PSR1257+12 may have begun as a binary of two stars of about $10 M_{\odot}$ with a period of a few days, and a planetary system around its centre of mass in which two planets were at distances 2.5-4 $\mathrm{AU}$. The binary evolved with mass transfer into a massive $\mathrm{X}$-ray binary. Such a system is not disrupted by the explosion that forms the neutron star because the exploding star has a low mass due to prior mass exchange. The velocity change of the binary due to the sudden mass loss and possibly an additional random kick imparted to the neutron star is typically not more than $40 \mathrm{~km}$ $\mathrm{s}^{-1}$, ensuring that a planet closer to a 20 $M_{\odot}$ binary than $10 \mathrm{AU}$ stays bound.

Next, the neutron star undergoes complete spiral-in into the core of the secondary, forming a so-called ThorneZytkow (TZ) object ${ }^{2}$. Such an object will expand and resemble a red supergiant near the end of its life. It will therefore engulf all planets closer than about 3-4 AU, causing their orbits to shrink and become (nearly) circular ${ }^{3,4}$. For example, for a planet at $3 \mathrm{AU}$, the spiral-in time, the remaining lifetime of the TZ object and the survival time of the planet in its envelope are similar, $10^{3}-10^{4} \mathrm{yr}$.

Many massive and intermediate-mass $\left(\sim 10 M_{\odot}\right)$ binaries with planetary systems could have at least one planet suited to our requirements. Also, as massive stars are often born in close binaries, a substantial fraction of pulsars are born in a possible progenitor system. The survival requirement then determines what fraction of pulsars actually retains one or more planets. We predict that pulsars can have both inner planets with orbital radii of about $0.2-3 \mathrm{AU}$ and outer planets with orbital eccentricities increasing with orbital radius. (The possible third planet $^{1}$ is close enough to have been in the TŻobject, but its orbit could be less completely circularized.)

The pulsar in our scheme has been embedded almost up to the end of the $T Z$ phase in a dense medium, accreting at the Eddington rate. Therefore, unlike models creating the planets from a slowly emptying disk, it can easily have been spun up to a period of $6 \mathrm{~ms}$. Its low magnetic field $\left(8.8 \times 10^{9} \mathrm{G}\right)$ may seem unexpected, but we know that field decay can be significant in descendants of massive binaries (for example the binary pulsars $1913+16$ and $0655+64)$. We considered models like the above involving a low-mass X-ray binary, because this is more commonly thought to be the site for forming recycled pulsars (see, however, ref. 5). We believe they can be excluded, because the planets would have to be much closer to the pulsar than is observed to have remained bound after the supernova explosion.
Because PSR $1257+12$ is very similar to other disk millisecond pulsars, this may indicate that some fraction of these formed from massive binaries. This has important implications for our understanding of binary evolution and would provide a possible solution to birth-rate problems of millisecond pulsars.

R. A. M. J. WIJERS

Princeton University Observatory,

Peyton Hall, Princeton,

New Jersey 08544, USA

E. P. J. VAN DEN HeUVEL M. H. VAN KERKWIJK

Astronomical Institute,

University of Amsterdam,

Kruislaan 403, 1098 SJ Amsterdam, The Netherlands

D. BHATTACHARYA

Raman Research Institute,

Bangalore 560 080, India

1. Wolszczan, A. \& Frail, D. A. Nature 355, 145-147
(1992).
2. Thorne, K. S. \& \&̇tkow, A. N. Astrophys. J. 212, 832-858
(1977).
3. Eggleton, P. P. \& Verbunt, F. Mon. Not. R. astr. Soc. $\mathbf{2 2 0}$,
13p-18p (1986).
4. Livio, M. \& Soker, N. Mon. Not. R. astr. Soc. 208,
763-781 (1984).
5. Blondin, J. M. \& Freese, K. Nature 323, 786 (1986).

\section{Mining in the Amazonian rainforests}

SIR - Although a sustainable agriculture or timber production is considered possible in some parts of the world ${ }^{1}$, such 'sustained' endeavours have failed in the Amazon region. On the other hand, mining in the Amazon is condemned by many environmentalists without any consideration of its positive aspects.

More than 100 million years of Amazonian history in equatorial latitudes, involving crustal bulging during the opening of the South Atlantic and subsequent continental sedimentation without any limestone formation, has resulted in thick laterites and soils poor in nutrients with a very low cationexchange capacity but high aluminium toxicity ${ }^{2}$. The intense tropical weathering produced depletion of silica and enrichment of certain metals, leading to accentuation of mineral reserves in the Brazilian Amazon ${ }^{2-4}$ (see table)

The prospects for the Amazonian ecosystem appear bleak. The main dangers emanate from large industrial projects, such as Grande Carajás; the construction of dozens of hydroelectric

\begin{tabular}{lrrr} 
& $\begin{array}{c}\text { Amazon } \\
\text { (reserves in }\end{array}$ & $\begin{array}{c}\text { Brazil } \\
\text { tons) }\end{array}$ & Global \\
\hline $\mathrm{Fe}$ & 18,000 & 36,000 & 210,900 \\
$\mathrm{Al}$ & 2,000 & 2,500 & 22,640 \\
$\mathrm{Mn}$ & 16 & 56 & 3,900 \\
$\mathrm{Nb}$ & 3.2 & 4.0 & 4.6 \\
$\mathrm{Sn}$ & $>0.48$ & 0.63 & 4.4 \\
$\mathrm{Au}$ & 0.013 & 0.033 & 0.070
\end{tabular}

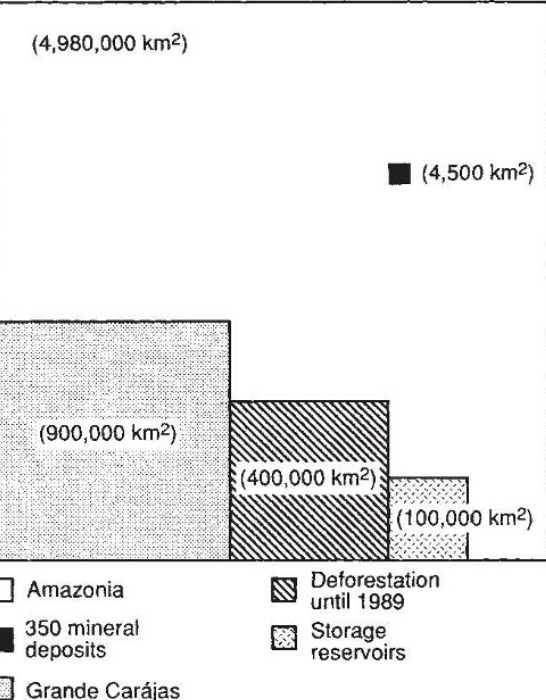

Mining would affect relatively small areas of Brazil's Amazonia compared to other possible uses of the land ${ }^{2.5}$.

power plants; the erroneous belief that large-scale exploitation of the forests and agronomic resources is a sustainable proposition; and the covert license to thousands of gold-diggers to spoil and pollute the environment with the use of mercury and production of waste dumps, as well as under-use of the resources by recovering less than 50 per cent of the metal. The possibility of any recovery may lie in an understanding of these dangers and/or political intervention such as the recent 'debt-for-natureswaps' agreement between Brazil and 